\title{
Direct Blue 2 diazo-boyarmaddesinin sulu çözeltiden P(HEMA) nanopartiküller ile uzaklaştırılması
}

\author{
Aslı GÖÇENOĞLU SARIKAYA* \\ Uludă̆ Üniversitesi Fen-Edebiyat Fakültesi Kimya Bölümü, Görükle Kampüsü, Bursa \\ Geliş Tarihi (Recived Date): 04.05.2018 \\ Kabul Tarihi (Accepted Date): 15.11.2018
}

\section{Özet}

Bu çallşmada, Direct Blue 2 (DB2) diazo-boyar maddesinin poli(Hidroksietil metakrilat) [P(HEMA)] nanopartikülleri ile sulu çözeltiden giderimi çalışılmıştır. Adsorpsiyona ortam pH'ının, sıcaklığın, başlangıç boya derişiminin ve temas süresinin etkileri incelenmiştir. Optimum koşullar altında, $50 \mathrm{mg} / \mathrm{mL}$ başlangıç boya derişimi ve $2 \mathrm{mg}$ polimer kullanılarak \% 89.1 giderim elde edilmiştir. Adsorpsiyon mekanizmasının anlaşılabilmesi için Langmuir, Freundlich ve Sips (Langmuir-Freundlich) izoterm sabitleri hesaplanmıştır. Elde edilen verilere göre adsorpsiyonun Langmuir izoterm modeline uygun olduğu $\left(R^{2}: 0.993, Q_{\max }: 27.1 \mathrm{mg} / \mathrm{g}\right)$ rapor edilmiştir. Adsorpsiyon öncesi ve sonrası boya derişimleri UV-Vis spektrofotometresinde ölçülmüş, adsorpsiyon öncesi ve sonrası polimer FTIR ve SEM ile karakterize edilmiştir.

Anahtar Kelimeler: Direct Blue 2, P(HEMA), adsorpsiyon, nanopartikül.

\section{Removal of diazo-dye Direct Blue 2 (DB2) in aqueus solution by $\mathrm{P}(\mathrm{HEMA})$ nanoparticles}

\begin{abstract}
In this work, the adsorption of diazo-dye Direct Blue 2 (DB2) in aqueus solution were worked by poly(hydroxyethyl methacrilate) [P(HEMA)]. The effect of $\mathrm{pH}$, temperature, initial dye concentration and contact time to adsorption were investigated. Under optimum conditions, $50 \mathrm{mg} / \mathrm{mL}$ initial dye concentration and $2 \mathrm{mg}$ of polymer were used and removal of dye was obtained as $89.1 \%$. To understand the adsorption mechanism, Langmuir, Freundlich and Sips (Langmuir-Freundlich) isotherm constants were calculated. According to the data obtained, it was reported that the adsorption was
\end{abstract}

\footnotetext{
*Aslı GÖÇENOĞLU SARIKAYA, agocenoglu@uludag.edu.tr, https://orcid.org/0000-0002-7161-7003
} 
suitable for Langmuir isotherm model $\left(R^{2}: 0.993, Q_{\max }: 27.1 \mathrm{mg} / \mathrm{g}\right)$. Concentrations of dye were measured before and after adsorption by $U V$-Vis spectrophotometer, polymer was characterized by FTIR and SEM before and after adsorption.

Keywords: Direct Blue 2, P(HEMA), adsorption, nanoparticle.

\section{Giriş}

Gelişen teknoloji ile birlikte tekstil, gıda [1], plastik, kozmetik, kağıt [2], deri, kauçuk ve farmasötik endüstrisinde yaygın bir şekilde kullanılan boyar maddeler çevre kirliliğine neden olmaktadır [3]. Özellikle tekstil fabrikalarından kaynaklı kirlilikler su kaynaklarını ya da yeraltı sularını kirleterek birçok farklı probleme yol açmaktadır. Yapılan çalışmalarda bu tür kirli suların karsinojenik, mutajenik, teratojenik ve toksik oldukları rapor edilmiştir [3]. Çevre kirliliğine neden olan bu tür boyalar metal ve klorür içeriklerinden dolayı suda yaşayan canlıların fotosentetik aktivitelerini etkilemektedir [4]. Bu tür boyalar sentetik olmaları ve kompleks aromatik moleküler yapılarından dolayı oldukça kararlıdırlar ve biyo-bozunur ya da foto-bozunur olmadıkları için bu tür yöntemlerle giderimleri kolay yapılamaz [5]. Atık sulardan boyar maddelerin giderimi için birçok farklı test ve uygulamanın yanı sıra yeni ve alternatif metodların geliştirildiği birçok makale mevcuttur. Bu yöntemler fiziksel, kimyasal veya biyolojik olabilir [6-8]. Anaerobik muamele, flotasyon, kimyasal koagülasyon, elektrokimyasal koagülasyon, elektrokinetik koagülasyon, geliştirilmiş oksidasyon prosesleri, membran filtrasyon, ozonlama, 1şınlama gibi teknikler yaygın olarak kullanılan yöntemlerdir [9-11]. Fakat bu yöntemlerin yüksek maliyetli olmaları, son ürün olarak toksik atık üretmeleri ve teknik açıdan bazı sınırlamaların olması gibi bazı dezavantajları vardır. Ayrıca bu tür yöntemler uygulamaya geçirildiğinde maliyet açısından maliyetli olmaları sebebiyle endüstri için cazip gelmemektedir. Bu nedenle, katı-sıvı ara yüzeyinde gerçekleşen fiziksel adsorpsiyon prosesi kirleticilerin uzaklaştırılmasında hem ekonomik hem de çevre dostu bir yöntem olarak kabul edilmektedir. Hem adsorpsiyon prosesinin maliyetinin ucuz olmasi, hem de adsorbentin ucuz ve rejenere edilebilir olması önemli bir avantaj sağlar [12-20].

Aktif karbon en yaygın kullanılan adsorbent olmasına rağmen [21], pahalı olması ve rejenerasyon maliyetinin yüksek olması nedeniyle bazı dezavantajları mevcuttur [22]. $\mathrm{Bu}$ tür kısitlamalardan dolayı daha ucuz ve kolay bulunabilen doğal ve atık materyallerin adsorben olarak kullanılabilirliği araştırılmaktadır. Kil [21-24], kitin [25], alg [26], tarım atıkları [27] ve lağım pisliği [28] gibi farklı materyallerin adsorbent olarak kullanılabilirliği bilim insanları tarafından rapor edilmiştir. Bunun yanı sıra karbon nanotüpler [29-31], nanopartiküller ve nanokompozitler [32-34] ve diğer ucuz adsorbentler [35-37] de sulu çözeltilerden boyaların adsorpsiyon yoluyla gideriminde kullanılan materyallerdir.

Boyalar, kimyasal yapılarına ya da kromoforlarına göre; azo, anthrakinon, fitalosiyanin, etan gibi farklı sınıflara ayrılır [38]. Tekstil boyar maddelerinden en yaygın kullanım alanı bulan azo boyaların kimyasal yapılarında bir veya daha fazla aromatik halka ve azo bağlı boya ajanı $(-\mathrm{N}=\mathrm{N}-)$ bulunmaktadır [39, 40]. Azo boyalar genel olarak kompleks, mutajenik, toksik ve kanserojen özelliktedirler [41]. Ayrıca bu tür azo boyar maddeler oldukça kararlı ve kimyasal ya da biyolojik proseslerle degradasyona karşı direnç göstermektedirler [42]. 
Direct Blue sınıfı boyar maddeler mono-, di- ve tri-azotlu olmak üzere üç ana sınıfa ayrılmaktadır. Bu tür boyalar toksik ve kanserojen [43] olmalarının yanı sıra bazı mikroorganizmaların direkt yıkımlarına ya da katalitik aktivitelerinin baskılanmasına yol açmaktadır [44]. Bu nedenle bu tür boyaların giderimi oldukça önemlidir. Direct Blue türü boyaların atık sulardan giderimi için farklı yöntemler kullanılmaktadır. Bu yöntemler son yıllarda araştırmacılar tarafından oksidasyon [45,46], fotokatalitik degradasyon [47], dekolorizasyon [48], elektrokoagülasyon [49] ve adsorpsiyon [50, 51, 52, 53] şeklinde rapor edilmiştir. Özellikle maliyetinin düşük olması, kolay hazırlanıyor olması ve uygulanan işlemin basit olması nedeniyle Direct Blue türü azo boyaların atık sulardan gideriminde adsorpsiyon yöntemi sıklıkla kullanılmaktadır [54, 55, 56, 57, 58]. Son yıllarda yapılan çalışmalarda buğday kabuğu [58], çok duvarlı karbon nanotüp [59], Carica papaya yaprağı [60], fıstık kabuğu atığı [61] ve modifiye zeolit [62] gibi farklı adsorbentlerin kullanılabilirliği rapor edilmiştir. Biyo-uyumlu ve ekonomik olan bu tür adsorbentlerin kullanılan adsorbent miktarının fazla olması [58] ve uzun adsorpsiyon süresi [58, 61], adsorpiyon kapasitesinin düşük olması [59, 60, 62] ve desorpsiyon ajanı olarak aseton gibi organik ürünlerin kullanılması [63] gibi bazı dezavantajları mevcuttur.

Bu çalışmada diazo-boyar madde olan Direct Blue 2'nin sulu çözeltilerden giderimi araştırılmıştır. Çalışmada adsorpsiyon yöntemi kullanılmış, adsorben madde olarak nano boyutlardaki poli(Hidroksietil metakrilat) [P(HEMA)] seçilmiştir. Biyo-uyumlu bir polimer olan P(HEMA) pH ve sıcaklığa karşı duyarlıdır ve suda kolaylıkla şişebilir. Dişçilik, oftalmik, ilaç salımı ve doku mühendisliği [64, 65] gibi farklı alanlarda kullanılan P(HEMA) aynı zamanda çevre dostu adsorben olarak su kirleticilerinin uzaklaştırılmasında da kullanılmaktadır [66]. Çift azo sınıfında olan Direct Blue 2'nin kimyasal yapısı Şekil 1'de verilmiştir. Farklı pH, süre, derişim ve sıcaklıklarda denemeler yapılarak Langmuir, Freundlich ve Langmuir-Freundlich izoterm modelleri uygulanmiştır.

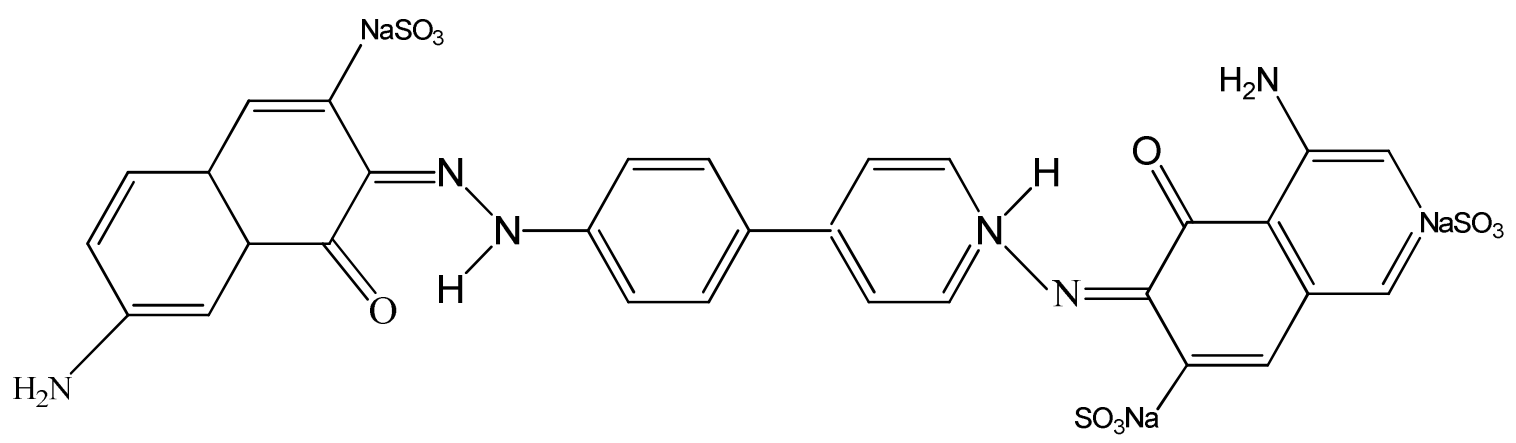

Şekil 1. Direct Blue 2'nin kimyasal yapısı.

\section{Materyal ve metot}

\subsection{Kullanilan kimyasallar}

Direct Blue 2 (DB2), hidroksietil metakrilat (HEMA; $\geq \% 99$ ), polivinil alkol (PVA; MA: 130 000) ve etilen glikol dimetakrilat (EGDMA; \% 98) Aldrich (Steinheim, Almanya)'ten sodyum dodesil sülfat (SDS; $\geq \%$ 98.5) Sigma (Steinheim, Almanya)'dan 
temin edilmiştir. Deneyde kullanılan diğer tüm kimyasal malzemeler analitik derecelidir.

\subsection{P(HEMA) nanopartiküllerin sentezi ve karakterizasyonu}

P(HEMA) nanopartiküllerin sentezi emülsiyon polimerizasyonu yöntemi ile gerçekleştirilmiştir. İlk olarak birinci sulu fazda PVA, SDS ve $\mathrm{NaHCO}_{3}$ çözünmüştür. İkinci sulu faz için PVA ve SDS ayrı bir yerde çözünmüştür. Yağ fazını hazırlamak için HEMA ve EGDMA çözülerek birinci sulu faza eklenmiş ve mini-emülsiyon hazırlanmıştır. Daha sonra hazırlanan mini-emülsiyon çözeltisi ikinci sulu faz ile birleştirilerek $\mathrm{NaHSO}_{3}$ ve amonyum persülfat eklenerek polimerizasyon gerçekleştirilmiştir. Polimerizasyon $40{ }^{\circ} \mathrm{C}$ ' de 6 saatte gerçekleştirilmiştir. Polimerizasyona girmemiş monomerlerin giderilmesi amaciyla polimerizasyon bittikten sonra sentezlenen nanopartiküller etanol ve su ile birkaç kez yıkanmıştır. Nanopartiküller yıkama işleminin ardından birkaç defa saf su ile yıkanmış ve saf su içerisinde muhafaza edilmiştir [67].

Sentezlenen nanopartiküllerin IR karakterizasyonu FTIR spektrofotometresi ile gerçekleştirilmiştir (Perkin Elmer Spectrum BX FTIR System). Nanopartiküllerin morfolojik karakterizasyonun yapılabilmesi için taramalı elektron mikroskopu (SEM) görüntüleri SEM2-Quanta 250FEG cihazı kullanılarak alınmıştır. Tüm spektrofotometrik ölçümler Perkin Elmer Lambda 35 UV/Vis Spektrometre ile gerçekleştirilmiştir.

\subsection{Adsorpsiyon çaltş̧malart}

Adsorpsiyon koşullarının belirlenebilmesi için farklı $\mathrm{pH}$, farklı sıcaklık ve farklı derişimlerde çalışmalar yapılmıştır. Adsorpsiyon çalışmaları DB2 ile farklı derişimlerde hazırlanan $(1,5,10,20,30,40,50,75$ ve $100 \mathrm{mg} / \mathrm{L})$ çözeltiden, farklı $\mathrm{pH}$ şartlarında $(3,4,5$, 6, 7 ve 8) gerçekleştirilmiştir. pH: 3-4-5 için asetat tamponu, pH: 67-8 için fosfat tamponu kullanılmıştır. Hazırlanan örnekler $125 \mathrm{rpm}$ çalkalama hızında olacak şekilde 1sitmalı inkübatöre konulmuştur. Adsorpsiyon çalışması sırasında örneklerin alınma sıklığı 0,10, 20, 30, 45, 60, 90, 120, 150 ve 180 dakika şeklinde olmuştur. Toplanan örnekler $10000 \mathrm{rpm}$ 'de 5 dakika santrifüjlenerek polimerlerin çökmesi sağlanmış, daha sonra renk ölçümü spektrofotometre $\left(\lambda_{\max } 568 \mathrm{~nm}\right)$ ile gerçekleştirilmiştir.

Çözeltide bulunan boya miktarının \% tutunma değeri $(\% R)$ (Eşitlik 1) ve adsorpsiyon kapasitesi $(q)$ (Eşitlik 2) hesaplanmıştır:

$\% R=\frac{\left(C_{o}-C_{e}\right)}{C_{o}} \times 100$

$q=\frac{\left(C_{o}-C_{e}\right) V}{m}$

Denklemde $C_{o}$ değeri başlangıç $\mathrm{DB} 2$ derişimini $(\mathrm{mg} / \mathrm{L}), C_{e}$ değeri dengedeki DB2 derişimini $(\mathrm{mg} / \mathrm{L}), V$ toplam hacmini $(\mathrm{mL}), m$ ise kullanılan adsorbent miktarını $(\mathrm{g})$ vermektedir.

\subsection{Adsorpsiyon İzotermleri}

Adsorbent yüzeyinde meydana gelen adsorpsiyon olay1, Langmuir, Freundlich ve Sips (Langmuir-Freundlich) izoterm modelleri ile açıklanmıştır. Langmuir izoterm modeli Eşitlik (3)'te gösterilmiştir [68]: 
$\frac{C_{e}}{q_{e}}=\frac{1}{Q_{L} K_{L}}+\frac{C_{e}}{Q_{L}}$

Burada $q_{e}$ adsorplanan DB2 miktarını $(\mathrm{mg} / \mathrm{g}), C_{e}$ dengedeki çözeltide adsorplanmadan kalan DB2 derişimini (mg/L), $Q_{L}$ maksimum adsorpsiyon kapasitesini $(\mathrm{mg} / \mathrm{g})$ ve $K_{L}$ adsorpsiyon enerjisi ile ilgili Langmuir sabitini göstermektedir. Deneysel sonuçlar $C_{e}$ 'ye karşı çizilen $C_{e} / q_{e}$ grafiği ile yukarıdaki denklemden yararlanılarak $Q_{L}$ ve $K_{L}$ değerleri hesaplanmıştır [69].

Heterojen yüzeyler için çok tabakalı adsorpsiyon modelinden türetilmiş Freundlich izoterm modeli Eşitlik (4)'te gösterilmiştir [70]:

$\ln q_{e}=\ln K_{F}+\frac{1}{n} \ln C_{e}$

$q_{e}$ denge anında polimer üzerinde adsorplanan DB2 miktarını $(\mathrm{mg} / \mathrm{g}), K_{F}$ adsorpsiyon kapasitesini $(\mathrm{mg} / \mathrm{g}), n$ Freundlich izoterm sabitini, $C_{e}$ denge halinde çözeltide kalan DB2 derişimini (mg/L) göstermektedir. Eşitlik (4)'teki $1 / n$ değeri sıfıra ne kadar yakınsa yüzey o kadar heterojen demektir [71].

Eşitlik (5)'te Sips izoterm modeline ait eşitlik verilmiştir. Bu izoterm modeli Langmuir ve Freundlich modellerinin birlikte geliştirilen bir kombinasyonudur [72].

$\frac{1}{q_{e}}=\frac{1}{Q_{\max } K_{s}}\left(\frac{1}{C_{e}}\right)^{1 / n}+\frac{1}{Q_{\max }}$

$q_{e}$ denge anında polimer üzerinde adsorplanan DB2 miktarını $(\mathrm{mg} / \mathrm{g}), K_{s}$ Sips izoterm modeli sabiti (L/mg), $n$ Sips modeli sabiti (mg), $Q_{\max }$ maksimum adsorpsiyon kapasitesini $(\mathrm{mg} / \mathrm{g}), C_{e}$ ise denge halinde çözeltide kalan DB2 derişimini $(\mathrm{mg} / \mathrm{L})$ göstermektedir.

\subsection{Desorpsiyon çalışmaları}

Nanopartiküllerin adsorpsiyon sonrası tekrar kullanımının araştırılması amacıyla desorpsiyon çalışmaları gerçekleştirilmiştir. $\mathrm{Bu}$ amaçla boya adsorbe edilmiş nanopartiküllerden boyanın geri alınması için pH 3 tampon çözeltisi hazırlanmış ve polimerler 3 saat boyunca tampon çözelti ile muamele edilmiştir. İşlem $5 \mathrm{kez}$ tekrarlanmış ve ortamda DB2 varlığı spektrofotometre ile takip edilmiştir.

\section{Sonuçlar ve tartışma}

\subsection{P(HEMA) Nanopartiküllerin Karakterizasyon}

Adsorpsiyon öncesi ve sonrası P(HEMA) nanopartiküllerin FTIR spektrumları alınmıştır. Şekil 2'de P(HEMA) nanopartikülüne ait FTIR spektrumu verilmiştir. Elde edilen verilere göre $3320 \mathrm{~cm}^{-1}$, de $(\mathrm{O}-\mathrm{H})$ gerilme titreşimi, $1716 \mathrm{~cm}^{-1}(\mathrm{C}=\mathrm{O})$ ve 2949 $\mathrm{cm}^{-1} \mathrm{CH}_{3}$ 'e ait $(\mathrm{C}-\mathrm{H})$ gerilmesi gözlenmiştir. $(\mathrm{C}-\mathrm{O})$ gerilme titreşimi ise $1268 \mathrm{~cm}^{-1}$ gözlenmiştir. DB2'nin yapıya adsorpsiyonu ile birlikte P(HEMA)'ya ait FTIR spektrumunda bazı değişiklikler olmuştur (Şekil 3). $3320 \mathrm{~cm}^{-1}$, de geniş bir bant olarak görülen $(\mathrm{O}-\mathrm{H})$ gerilme titreşimi, yapıya DB2'nin adsorbe olmasıyla kaybolmuştur. Bunun nedeni olarak boya ve polimer arasındaki olası H-bağı gösterilebilir. 
Nanopartiküllerin morfolojik özelliklerinin belirlenebilmesi için SEM görüntüleri alınmıştır (Şekil 4). Elde edilen görüntülere göre, P(HEMA) partiküllerin nano-boyutta oldukları, yüzeylerinin düzgün ve küresel formda oldukları görülmektedir.

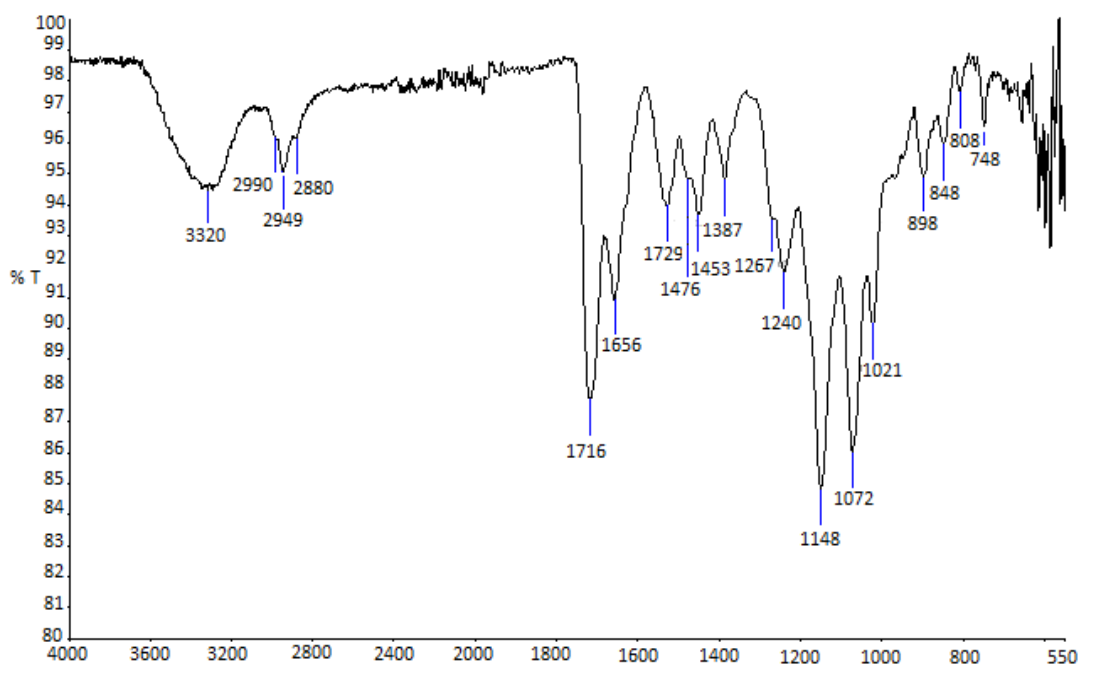

Şekil 2. P(HEMA) nanopartiküllerin FTIR spektrumu.

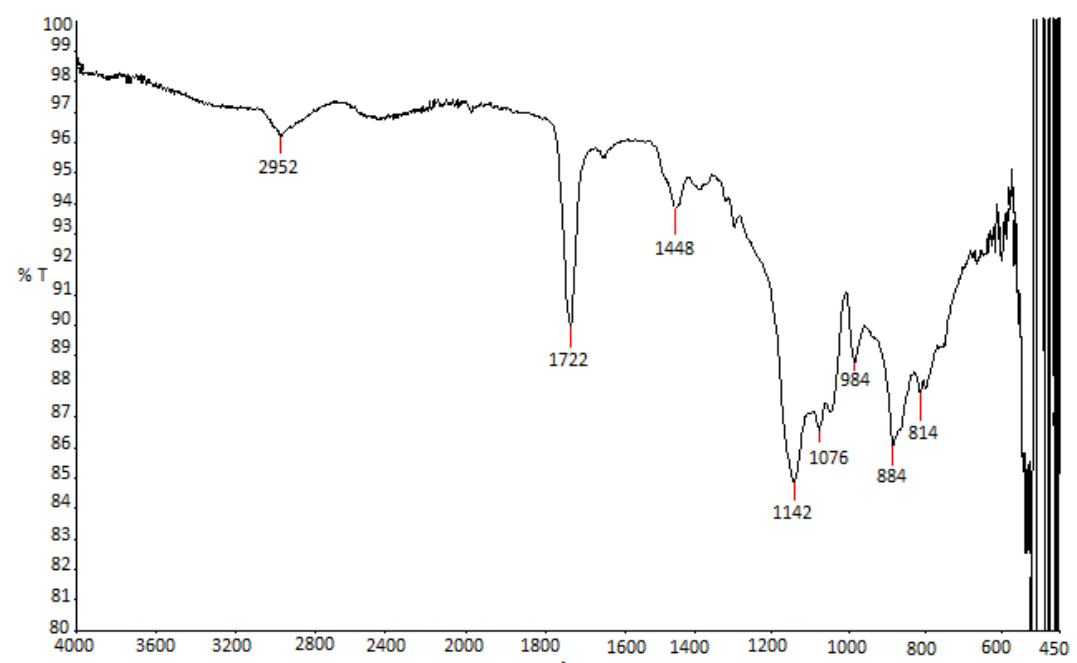

Şekil 3. DB2 adsorbe olmuş P(HEMA) nanopartiküllerin FTIR spektrumu.

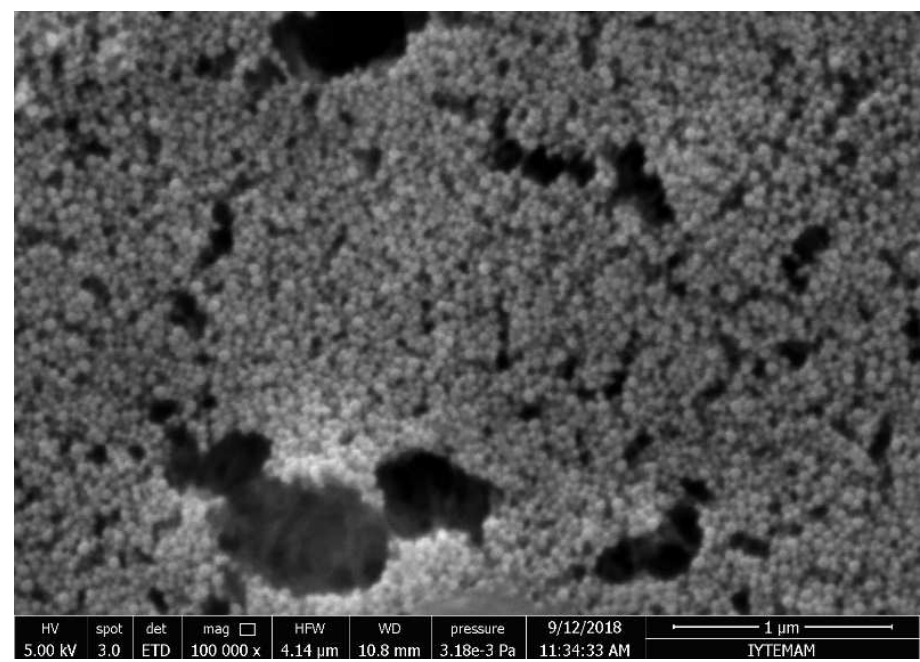

Şekil 4. P(HEMA) nanopartiküllerin SEM görüntüsü. 


\subsection{Adsorpsiyona pH'ın etkisi}

$\mathrm{pH}$ 3-8 arası farklı $\mathrm{pH}$ değerlerinde hazırlanan DB2 çözeltilerinin her birine $2 \mathrm{mg}$ nanopartikül eklenerek $q$ adsorpsiyon kapasitesi ve $\% R$ tutunma değeri hesaplanmıştır. Adsorpsiyon proseslerinde çözeltinin $\mathrm{pH}$ değeri, adsorbanın yükünü ve adsorpsiyon kapasitesini etkileyen önemli bir parametredir [73]. Çözeltinin pH'ı adsorbent yüzey yükünü, adsorblanan maddenin iyonizasyonunu ve adsorbentin aktif gruplarının iyonizasyonunu etkileyebilmektedir. Bu nedenle, boya giderimi proseslerinde ortam pH'1 oldukça önemlidir [74]. Tablo 1'de farklı tekstil boyar maddelerinin farkl1 pH değerlerindeki \% giderim değerleri verilmiştir. $\mathrm{pH}$ değeri değiştikçe boyar maddelerin adsorbentlere tutunma değerleri de değişmektedir. Anyonik bir boya olan DB2'nin elektrostatik özelliği farklı $\mathrm{pH}$ değerlerinde değişmektedir. $\mathrm{pH}$ 3-8 tamponları ile hazırlanan DB2'nin adsorpsiyon kapasitesi $(10.86 \mathrm{mg} / \mathrm{g})$ ve \% tutunma değeri (\% 61.54) karşılaştırıldığında en yüksek değerin $\mathrm{pH}$ 7'de gerçekleştiği gözlenmiştir. Adsorpsiyona pH'ın etkisi Şekil 5'te gösterilmiştir. Elde edilen bulgulara göre elektrostatik etkileşimin adsorpsiyon kapasitesi üzerinde etkili olduğu düşünülmektedir.

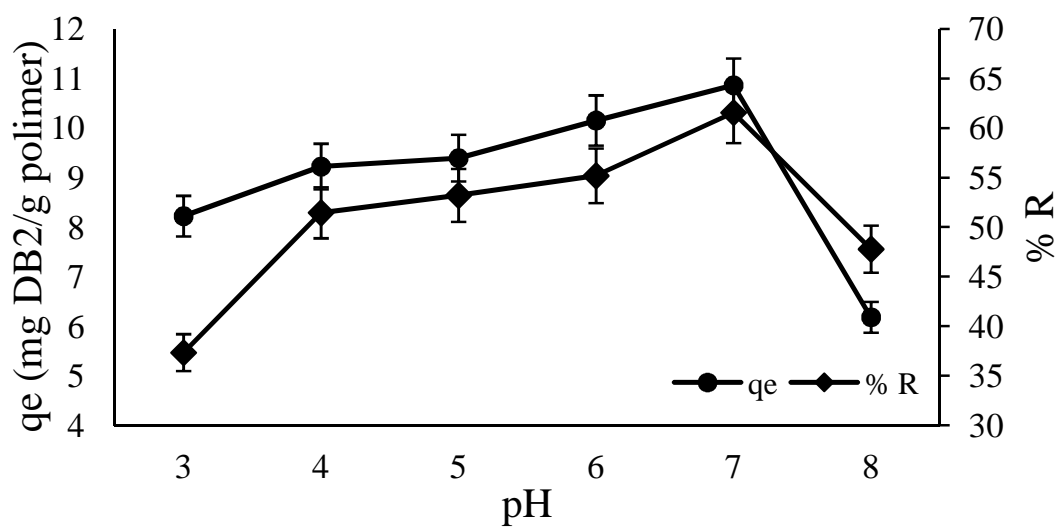

Şekil 5. DB2'nin adsorpsiyonuna pH'1n etkisi.

Tablo 1. Farklı adsorbatların \% giderim miktarının karşılaştırılması.

\begin{tabular}{|c|c|c|c|c|}
\hline Adsorbent & Adsorbat & $\begin{array}{c}\mathrm{pH} \\
\text { aralığı }\end{array}$ & $\% \mathrm{R}$ aralı̆̆ & Referans \\
\hline Modifiye alümina & Kristal menekşe & $2.6-10.8$ & $20-80$ & 75 \\
\hline Aktif kil & Metil mavisi & $2-9$ & $60-95$ & 76 \\
\hline Kaolin & Kristal menekşe & $2-7$ & $65-95$ & 77 \\
\hline Kül & Metil mavisi & $2-8$ & $36-45$ & 78 \\
\hline Çam yaprağı & Metil mavisi & $2-11$ & $20-80$ & 79 \\
\hline $\mathrm{Fe}_{2} \mathrm{O}_{3}$ & Asit kırmızısı 27 & $1.5-10.5$ & $98-28$ & 80 \\
\hline Aktif karbon & Direct Blue 86 & $1-8$ & $35-61$ & 81 \\
\hline Kül & Blue 5 & 10.46 & $25.58-34.30$ & 82 \\
\hline $\begin{array}{l}\text { Biyo-hidrojen fermentasyon atığından } \\
\text { türetilmiş katif karbon }\end{array}$ & $\begin{array}{l}\text { Direct Navy Blue } \\
106\end{array}$ & $2-10$ & $0-96$ & 83 \\
\hline $\begin{array}{c}\text { Ficus carica' dan türetilmiş aktif } \\
\text { karbon }\end{array}$ & Metil mavisi & $2-12$ & $12-86$ & 84 \\
\hline $\begin{array}{l}\text { Poli(glisidilmetakrilat) temelli } \\
\text { manyetik silika partikülleri }\end{array}$ & Direct Blue 6 & $2-9$ & 87 & 50 \\
\hline $\begin{array}{l}\text { Poli(glisidilmetakrilat) temelli } \\
\text { manyetik silika partikülleri }\end{array}$ & Direct Black 38 & $2-9$ & 64 & 50 \\
\hline $\mathrm{Kitosan} / \mathrm{SiO}_{2} / \mathrm{karbon}$ nanotüp & Direct Blue 71 & $2-10$ & $80-89$ & 85 \\
\hline Kitosan/ $/ \mathrm{SiO}_{2} /$ karbon nanotüp & Reaktif Blue 19 & $2-10$ & $63-98$ & 85 \\
\hline Modifiye zeolit & Direct Blue 129 & $2-8$ & $79-100$ & 86 \\
\hline Peroksidaz & Direct Blue 2 & $3-7.5$ & $21-75 \pm 4$ & 87 \\
\hline Kaolin & Metil mavisi & $2-10$ & $68-98$ & 88 \\
\hline P(HEMA) & Direct Blue 2 & 3-8 & 37.86-89.05 & $\begin{array}{l}\text { Mevcut } \\
\text { çalışma }\end{array}$ \\
\hline
\end{tabular}




\subsection{Adsorpsiyona başlangıç boya derişiminin etkisi}

Adsorban olarak P(HEMA) nanopartiküller kullanılarak DB2 adsorpsiyonunda boya derişiminin etkisi Şekil 6'da gösterilmiştir. DB2'nin derişiminin $1 \mathrm{mg} / \mathrm{L}$ 'den 100 mg/L'ye çıkmasıyla adsorplama kapasitesi 1.43 mg/g'dan $14.32 \mathrm{mg} / \mathrm{g}$ 'a yükselirken, \% tutunma miktarı azalma göstermektedir. Başlangıç boya derişiminin artması ile adsorplanan madde miktarı da doğru orantılı olarak artış göstermektedir. Başlangıç boya derişiminin artması, adsorban ve boya arasındaki etkileşimin de artmasına yol açar. Fakat artan boya derişimiyle polimerin adsorpsiyon kapasitesinin dolması sonucu daha fazla adsorpsiyon gerçekleşmez ve bu nedenle boyanın \% tutunma miktarı artan derişimle birlikte düşer. \% tutuma miktarı düşük derişimde \% 84.39 iken derişimin arttırılmasıyla \% 37.86' ya kadar düşmüştür.

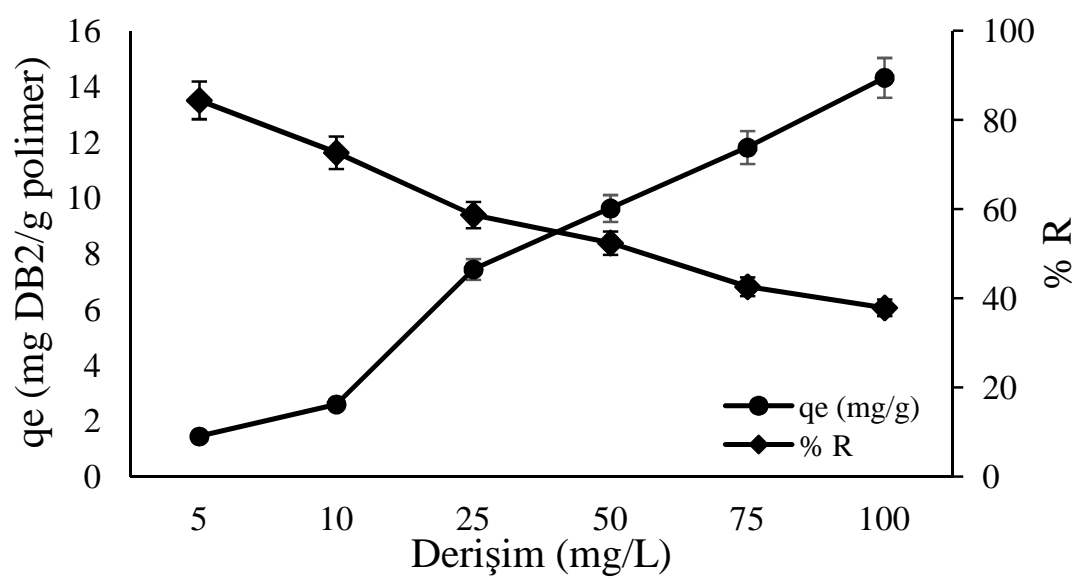

Şekil 6. Başlangıç boya derişiminin adsorpsiyon kapasitesi (qe) ve \% tutunma miktarı $(\% \mathrm{R})$ üzerine etkisi.

\subsection{Sıcaklı̆̆ın Adsorpsiyona Etkisi}

Ortam sıcaklığının adsorpsiyona etkisini incelemek için $4{ }^{\circ} \mathrm{C}, 25{ }^{\circ} \mathrm{C}$ ve $45^{\circ} \mathrm{C}$ 'de çalışılmıştır. Denge durumundayken sıcaklığın adsorpsiyon kapasitesi (Şekil 7a) ve \% tutunma miktarı üzerindeki etkisi (Şekil 7b) verilmiştir. Elde edilen sonuçlara göre sıcaklık arttıkça qe değeri ve \% $\mathrm{R}$ değeri artmaktadır. Bunun nedeni olarak, boyanın artan yüzey aktivitesi ve artan kinetik enerjisi gösterilebilir [89].

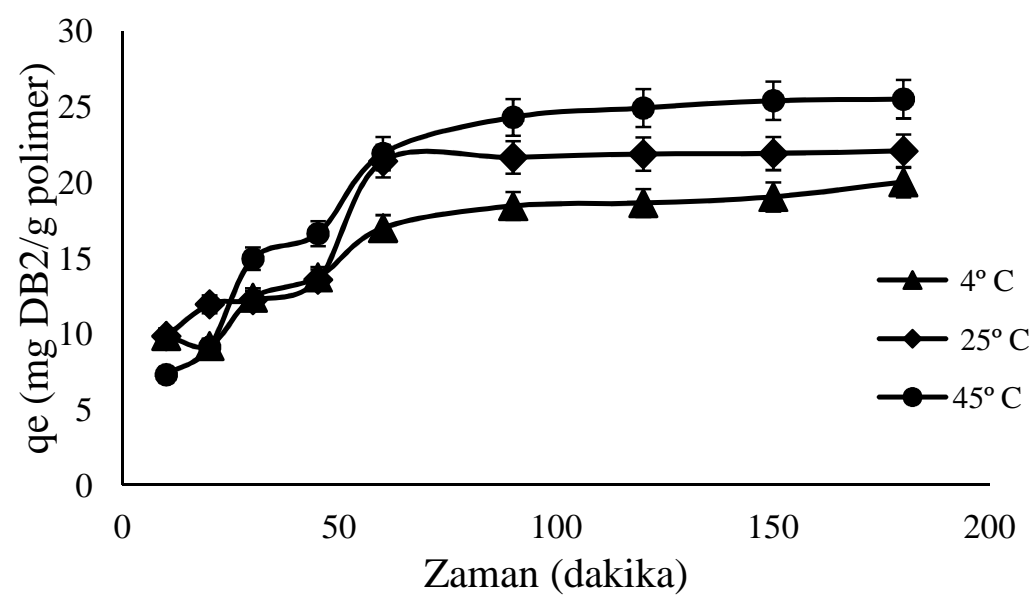

(a) 


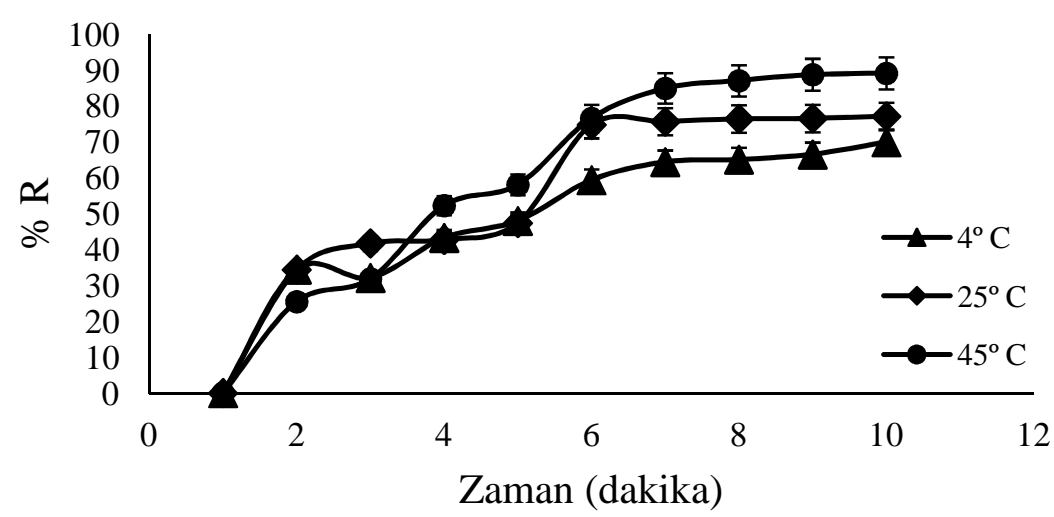

(b)

Şekil 7. Sıcaklığın (a) adsorpsiyon kapasitesi (qe) ve (b) \% tutunma miktarı (\% R) üzerine etkisi.

\subsection{Adsorban miktarının adsorpsiyona etkisi}

Adsorban olarak kullanılan P(HEMA) nanopartikül miktarının adsorpsiyona etkisini incelemek için 2, 4, 8, 20 ve 40 mg polimer miktarı kullanılmıştır. Deney koşulları 25 ${ }^{\circ} \mathrm{C}$ 'de $50 \mathrm{mg} / \mathrm{mL}$ başlangıç boya derişimi olarak ayarlanmıştır. Denge durumundayken adsorban miktarının adsorpsiyon kapasitesi ve \% tutunma miktarı üzerindeki etkisi (Şekil 8) verilmiştir. Elde edilen sonuçlara göre adsorban miktarı arttıkça qe değeri ve $\% \mathrm{R}$ değeri artmaktadır.

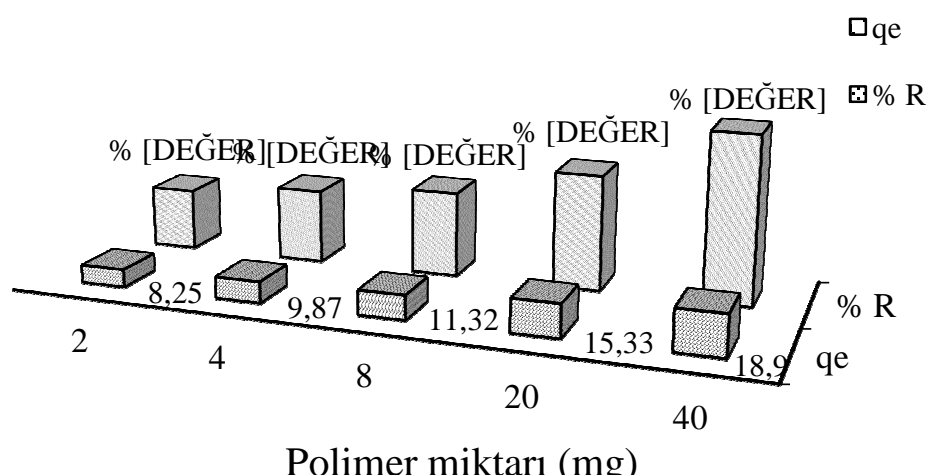

Şekil 8. Adsorban miktarının adsorpsiyon kapasitesi (qe) ve \% tutunma miktarı (\% R) üzerine etkisi.

\subsection{Adsorpsiyon izotermleri}

P(HEMA) nanopartiküller kullanılarak DB2'nin adsorpsiyonu için Langmuir, Freundlich ve Sips (Langmuir-Freundlich) izoterm modelleri uygulanmış ve Tablo 2'de Langmuir izotermi için $Q_{L}$ ve $K_{L}$ değerleri, Freundlich izotermi için $K_{f}$ ve $n$ değerleri, Sips izotermi için $Q_{\max }$ ve $K_{S}$ değerleri ve her üç izoterm için regresyon katsayıları $\left(\mathrm{R}^{2}\right)$ verilmiștir.

Langmuir izoterm modeli, uniform ve sınırlı adsorpsiyon alanına sahip tek tabakalı adsorpsiyon modelidir. P(HEMA)'nın DB2 için en yüksek adsorpsiyon kapasitesi 
$14.31 \mathrm{mg} / \mathrm{g}, \mathrm{K}_{\mathrm{L}}$ değeri ise 0.011 olarak hesaplanmıştır. Freundlich izoterm modelinde ise adsorpsiyon kapasitesi $\left(K_{f}\right)$ ve heterojenite faktörü $(1 / n)$ değerleri hesaplanmıştır. $1 / n$ değeri 0-1 aralığındadır. $1 / n$ faktörü 0'a ne kadar yakınsa yüzey o kadar heterojendir. P(HEMA)'nın DB2 adsorpsiyonuna ait $1 / n$ değeri $0.23, K_{S}$ değeri ise 0.34 olarak bulunmuştur. $1 / n$ değerinin 1 'den küçük olması adsorbanların boya giderimi için uygun olduğunun bir göstergesidir [90].

P(HEMA)'nın DB2 adsorpsiyonu için $\mathrm{R}^{2}$ değerleri hesaplandığında Langmuir ve Freundlich izotermlerinin uygun olduğu fakat Langmuir izoterm modeline daha fazla uyumlu olduğu görülmektedir. Tablo 2'de görüldüğü gibi $\mathrm{R}^{2}$ değerleri Langmuir, Freundlich ve Sips izoterm modelleri için sırasıyla 0.99 , 0.97 ve 0.92 'dir.

Tablo 2. Langmuir, Freundlich ve Sips izoterm modellerinin parametreleri.

\begin{tabular}{|c|c|c|c|c|c|c|c|c|c|}
\hline \multirow[b]{2}{*}{$\begin{array}{l}\text { Sicaklık } \\
\text { (K) }\end{array}$} & \multicolumn{3}{|c|}{$\begin{array}{l}\text { Langmuir İzoterm } \\
\text { Değerleri }\end{array}$} & \multicolumn{3}{|c|}{$\begin{array}{l}\text { Freundlich İzoterm } \\
\text { Değerleri }\end{array}$} & \multicolumn{3}{|c|}{ Sips İzoterm Değerleri } \\
\hline & $\begin{array}{c}K_{L} \mathrm{X} \\
10^{2} \\
(\mathrm{~L} / \mathrm{mg})\end{array}$ & $\begin{array}{c}Q_{L} \\
(\mathrm{mg} / \mathrm{g})\end{array}$ & $\mathrm{R}^{2}$ & $\begin{array}{c}K_{F} \\
(\mathrm{~L} / \mathrm{mg})\end{array}$ & $n$ & $\mathrm{R}^{2}$ & $\begin{array}{c}K_{S} \mathrm{X} \\
10^{2} \\
(\mathrm{~L} / \mathrm{mg})\end{array}$ & $\begin{array}{c}Q_{\max } \\
(\mathrm{mg} / \mathrm{g})\end{array}$ & $\mathrm{R}^{2}$ \\
\hline 298 & 1.08 & 27.1 & 0.993 & 0.423 & 4.267 & 0.975 & 34.3 & 1.644 & 0.921 \\
\hline
\end{tabular}

\subsection{Desorpsiyon çalışmalart}

DB2'nin P(HEMA)'dan desorpsiyonu $\mathrm{pH}$ : 3 asetat tamponu ile yapılmıştır. Adsorpsiyon-desorpsiyon döngüsü beş kere tekrarlanmış ve DB2 adsorpsiyon kapasitesinin değişmediği görülmüştür $(10.86,10.75,10.53,10.60$ ve $10.03 \mathrm{mg} \mathrm{DB} 2 / \mathrm{g}$ polimer). Elde edilen sonuçlara göre, P(HEMA) nanopartiküllerinin, adsorpsiyon kapasitesinde ciddi bir kayıp olmadan sulu çözeltilerden DB2'nin adsorpsiyonunun defalarca yapılabileceğini göstermektedir.

\section{Tartışma}

$\mathrm{Bu}$ çalışmada, DB2 di-azo boyarmaddesinin sulu çözeltilerden P(HEMA) nanopartiküller ile adsorpsiyonu çalışılmıştır. Adsorpsiyona $\mathrm{pH}$ etkisi, temas süresi ve farklı derişimlerdeki DB2 çözeltisinin etkileri incelenmiştir. Di-azo yapıda olan boya pH 7'de en yüksek adsorplama kapasitesi göstermiştir. Temas süresi arttıkça, adsorbanın boyayı tutma kapasitesi de artmaktadır. DB2 derişimi arttıkça adsorpsiyon kapasitesi de $1.44 \mathrm{mg} / \mathrm{g}$ ' dan $14.43 \mathrm{mg} / \mathrm{g}$ 'a yükselmektedir. Fakat derişim arttıkça boyanın çözelti ortamından \% tutunma miktarı azalmaktadır. Bunun nedeni, boya derişiminin artmasına bağlı olarak polimerin adsorplama kapasitesinin dolması nedeniyle \% tutunma miktarının düşmesidir. Temas süresi ve sıcaklık arttıkça adsorpsiyon kapasitesi ve \% tutunma miktarı doğrusal olarak artmıştır. Sicaklığın adsorpsiyon kapasitesi ve \% tutunma miktarına olan etkisini incelemek için $4{ }^{\circ} \mathrm{C}, 25^{\circ} \mathrm{C}$ ve $45^{\circ} \mathrm{C}^{\prime}$ de çalışılmış ve qe miktarının $20.03 \mathrm{mg} / \mathrm{g}$ 'dan $25.51 \mathrm{mg} / \mathrm{g}$ 'a arttığ 1 , \% R'nin ise \% 69.91'den \% 89.05'e yükseldiği gözlenmiştir. Aynı zamanda $45^{\circ} \mathrm{C}$ 'deki qe ve \% $\mathrm{R}$ değerlerine bakıldığında, adsorpsiyonun 10. dakikasında qe $7.95 \mathrm{mg} / \mathrm{g}$ iken 90. dakikada $24.31 \mathrm{mg} / \mathrm{g}, 180$. dakikada ise bu değer $25.51 \mathrm{mg} / \mathrm{g}$ olarak bulunmuştur. Elde edilen bu sonuca göre, temas süresi arttıkça $\mathrm{q}_{\mathrm{e}}$ değeri de artmaktadır. Boyanın polimer tarafından adsorplanması ilk 90 dakikada hızlı bir şekilde artarken, 90. dakikadan sonra değişiklik göstermemektedir. Adsorpsiyonun doğasının aydınlatılabilmesi için izoterm çalışması yapılmıştır. Bunun için Langmuir, Freundlich ve Sips izoterm modelleri incelenmiştir. Elde edilen verilere göre, adsorpsiyonun Langmuir izoterm modeline 
daha uygun olduğu, adsorpsiyonun tek tabakalı, uniform ve sınırlı adsorpsiyon alanına sahip olduğu rapor edilmiştir. Kullanılan polimerin tekrar kullanılabilirliğinin araştırılması için adsorpsiyon-desorpsiyon çalışmaları yapılmıştır. Desorpsiyon için pH 3 tampon çözeltisi kullanılmış ve adsorpsiyon-desorpsiyon döngüsü aynı polimer ve boya çözeltisi ile 5 kere tekrarlanmıştır. Elde edilen sonuçlara göre, kullanılan polimerin adsorplama kapasitesinde çok fazla değişiklik gözlenmeden defalarca kullanılabileceği saptanmıştır.

\section{Teşekkür}

$\mathrm{Bu}$ çalışmanın gerçekleşmesi esnasında yardımlarını esirgemeyen Prof. Dr. Nurdan Kaşıkara Pazarlığlu, Doç. Dr. Alper Akkaya ve Öğr. Gör. Dr. Emre Erden'e teşekkür ederim. Bu çalışmanın tamamı Ege Üniversitesi Fen Fakültesi Biyokimya Bölümü’nde gerçekleştirilmiştir.

\section{Kaynaklar:}

[1] Rajabi, M., Mirza, B., Mahanpoor, K., Mirjalili, M., Najafi, F., Moradi, O., Sadegh, H., Shahryari-ghoshekandi, R., Asif, M., Tyagi, I., Agarwal, S. ve Gupta, V. K., Adsorption of malachite green from aqueous solution by carboxylate group functionalized multi-walled carbon nanotubes: Determination of equilibrium and kinetics parameters, Journal of Industrial and Engineering Chemistry, 34, 130-138, (2016).

[2] Kaur, S. ve Rani, R. K., Adsorption kinetics fort he removal of hazardous dye congo red by biowaste materials as adsorbents Mahajan, Journal of Chemistry, $1-12,(2013)$.

[3] García, E. R., Medina, R. L., Lozano, M. M., Pérez, I. H., Valero, M. J. ve Franco, A. M. M., Adsorption of Azo-Dye Orange II from Aqueous Solutions Using a Metal-Organic Framework Material: Iron- Benzenetricarboxylate, Materials, 7, 8037-8057, (2014).

[4] Hameed, B. H. ve El-Khaiary, M. I., Equilibrium, kinetics and mechanism of malachite green adsorption on activated carbon prepared from bamboo by $\mathrm{K}_{2} \mathrm{CO}_{3}$ activation and subsequent gasification with $\mathrm{CO}_{2}$, Journal of Hazardous Materials, 157, 2-3, 344-351, (2008).

[5] Chen, H. ve Zhao, J., Adsorption study for removal of Congo red anionic dye using organo-attapulgite, Adsorption, 15, 4, 381-389, (2009).

[6] Hamoda, M. F., Al-Ghusain, I. ve Al-Mutairi, N. Z., Sand filtration of wastewater for tertiary treatment and water reuse, Desalination, 164, 203-211, (2004).

[7] Shi, B. Y., Li, G. H., Wang, D. S., Feng, C. H. ve Tang, H. X., Removal of direct dyes by coagulation: the performance of preformed polymeric aluminum species, Journal of Hazardous Materials, 143, 567-574, (2007).

[8] Slokar, Y. M. ve Lemarechal, A. M., Methods of decoloration of textile wastewaters, Dyes Pigments, 37, 335-356, (1998).

[9] Kornaros, M. ve Lyberatos, G., Biological treatment of wastewaters from a dye manufacturing company using a trickling filter, Journal of Hazardous Materials, 136, 95-102, (2006).

[10] Hall, K., Eagleton, L., Acrivos, A., ve Vermeulen, T., Pore-and solid-diffusion kinetics in fixed-bed adsorption under constant pattern conditions, Industrial \& Engineering Chemistry Fundamentals, 5, 212-223, (1966). 
[11] Kaur, S., Rani, S., ve Mahajan, R. K., Adsorption kinetics fort he removal of hazardous dye congo red by biowaste materials as adsorbents, Journal of Chemistry, 2013, 1-12, (2013).

[12] Abramian, L. ve El-Rassy, H., Adsorption kinetics and thermodynamics of azodye Orange II onto highly porous titania aerogel, Chemical Engineering Journal, 150, 403-410, (2009).

[13] Ma, J., Qi, J., Yao, C., Cui, B., Zhang, T. ve Li, D., A novel bentonite-based adsorbent for anionic pollutant removal from water, Chemical Engineering Journal, 200, 97-103, (2012).

[14] Ribeiro, R. S., Fathy, N. A., Attia, A. A., Silva, A. M. T., Faria, J. L. ve Gomes, H. T., Activated carbon xerogels for the removal of the anionic azo dyes Orange II and Chromotrope 2R by adsorption and catalytic wet peroxide oxidation, Chemical Engineering Journal , 195, 112-121, (2012).

[15] Hsiu-Mei, C., Ting-Chien, C., San-De, P. ve Hung-Lung, C., Adsorption characteristics of Orange II and Chrysophenine on sludge adsorbent and activated carbon fibers, Journal of Hazardous Materials, 161, 1384-1390, (2009).

[16] Rodríguez, A., García, J., Ovejero, G. ve Mestanza, M., Adsorption of anionic and cationic dyes on activated carbon from aqueous solutions: Equilibrium and kinetics, Journal of Hazardous Materials, 172, 1311-1320, (2009).

[17] Chiou, M. S., Ho, P. Y. ve Li, H. Y., Adsorption of anionic dyes in acid solutions using chemically cross-linked chitosan beads, Dyes Pigments, 60, 6984, (2004).

[18] Güzel, F., Saygılı, H., Saygılı, G. A. ve Koyuncu, F., Elimination of anionic dye by using nanoporous carbon prepared from an industrial biowaste, Journal of Molecular Liquids, 194, 130-140, (2014).

[19] Ayoub, G. M., Hamzeh, A. ve Semerjian, L., Post treatment of tannery wastewater using lime/bittern coagulation and activated carbon adsorption, Desalination, 273, 359-365, (2011).

[20] Papadia, S., Rovero, G., Fava, F. ve Di Gioia, D., Comparison of different pilot scale bioreactors for the treatment of a real wastewater from the textile industry, International Biodeterioration \& Biodegradation, 65, 396-403, (2011).

[21] Ozcan, A. ve Ozcan, A. S., Adsorption of Acid Red 57 from aqueous solutions onto surfactant-modified sepiolite, Journal of Hazardous Materials, 125, 1-3, 252-259, (2005).

[22] Sílvia C. R. ve Santos Rui, A. R., Boaventura Adsorption modelling of textile dyes by sepiolite, Applied Clay Science, 42, 137-145, (2008).

[23] Ozcan, A. S. ve Ozcan, A., Adsorption of acid dyes from aqueous solutions onto acid-activated bentonite, Journal of Colloid and Interface Science, 276, 1, 3946, (2004).

[24] Ozdemir, O., Armagan, B., Turan, M. ve Celik, M. S., Comparison of the adsorption characteristics of azo-reactive dyes on mesoporous minerals, Dyes and Pigments, 62, 1, 49-60, (2004).

[25] Wibulswas, R., Batch and fixed bed sorption of methylene blue on precursor and QACs modified montmorillonite, Separation and Purification Technology, 39, 1-2, 3-12, (2004).

[26] Figueiredo, S. A., Loureiro, J. M. ve Boaventura, R. A., Natural waste materials containing chitin as adsorbents for textile dyestuffs: batch and continuous studies, Water Research, 39, 17, 4142-4152, (2005). 
[27] Aksu, Z. ve Tezer, S., Equilibrium and kinetic modelling of biosorption of Remazol Black B by Rhizopus arrhizus in a batch system: effect of temperature, Process Biochemistry, 36, 5, 431-439, (2000).

[28] Robinson, T., Chandran, B. ve Nigam, P., Removal of dyes from an artificial textile dye effluent by two agricultural waste residues, corncob and barley husk, Environment International, 28, 1-2, 29-33, (2002).

[29] Mahmoodian, H., Moradi, O., Shariatzadeha, B., Saleh, T. A., Tyagi, I., Maity, A., Asif, M. ve Gupta, V. K., Enhanced removal of methyl orange from aqueous solutions by poly HEMA-chitosan-MWCNT nano-composite, Journal of Molecular Liquids, 202, 189-198, (2014).

[30] Saleh, T. A. ve Gupta, V. K., Column with CNT/magnesium oxide composite for lead(II) removal from water, Environmental Science and Pollution Research, 19, 1224-1229, (2012).

[31] Nekouei, F., Nekouei, S., Tyagi, I. ve Gupta, V. K., Kinetic, thermodynamic and isotherm studies for acid blue 129 removal from liquids using copper oxide nanoparticle-modified activated carbon as a novel adsorbent, Journal of Molecular Liquids, 201, 124-133 (2015).

[32] Gupta, V. K., Jain, R., Mittal, A., Agarwal, S. ve Sikarwar, S., Photo-catalytic degradation of toxic dye amaranth on $\mathrm{TiO}(2) / \mathrm{UV}$ in aqueous suspensions, Material Science and Engineering C, 32, 12-17, (2012).

[33] Gupta, V. K. ve Nayak, A., Cadmium removal and recovery from aqueous solutions by novel adsorbents prepared from orange peel and $\mathrm{Fe} 2 \mathrm{O} 3$ nanoparticles, Chemical Engineering Journal, 180, 81-90, (2012).

[34] Gupta, V. K., Jain, R., Agarwal, S. ve Shrivastava, M., Removal of the hazardous dye-Tartrazine by photodegradation on titanium dioxide surface, Material Science \& Engineering C, 31, 1062-1067, (2011).

[35] Gupta, V. K., Mittal, A. ve Mittal, J., Removal and recovery of chrysoidine Y from aqueous solutions by waste materials, Journal of Colloid and Interface Science, 344, 497-507, (2010).

[36] Gupta, V. K., Srivastava, S. K., Mohan, D. ve Sharma, S., Design parameters for fixed bed reactors of activated carbon developed from fertilizer waste for the removal of some heavy metal ions, Waste Management, 17, 517-522, (1998).

[37] Gupta, V. K., Mittal, A. ve Mittal, J., Decoloration treatment of a hazardous triarylmethane dye, Light Green SF (Yellowish) by waste material adsorbents, Journal of Colloid and Interface Science, 342, 518-527, (2010).

[38] Movahedian Attar, H. ve Rezaee, R., Investigating the efficiency of advanced photochemical oxidation (APO) technology in degradation of direct azo dye by $\mathrm{UV} / \mathrm{H}_{2} \mathrm{O}_{2}$ process, Water and Wastewater, 59, 75-83, (2006).

[39] Peng, Y., Fu, D., Liu, R., Zhang, F. ve Liang, X., $\mathrm{NaNO}(2) / \mathrm{FeCl}(3)$ catalyzed wet oxidation of the azo dye Acid Orange 7, Chemosphere, 71, 5, 990-997, (2008).

[40] Bali, U., Catalkaya, E. ve Sengul, F., Photodegradation of Reactive Black 5, Direct Red 28 and Direct Yellow 12 using UV, UV/ $\mathrm{H}_{2} \mathrm{O}_{2}$ and $\mathrm{UV} / \mathrm{H}_{2} \mathrm{O}_{2} / \mathrm{Fe}^{2+}$ : A comparative study, Journal of Hazardous Materials, 114, 1-3, 159-66, (2004).

[41] Bazrafshan, E., Mostafapour, F.K. ve Mahvi, A. H., Phenol removal from aqueous solutions using Pistachio-nut shell ash as a low cost adsorbent, Fresenius Environmental Bulletin, 21, 10, 2962-2968, (2012).

[42] Liu, Y., Chen, X., Li, J. ve Burda, C., Photocatalytic degradation of azo dyes by nitrogen-doped TiO2 nanocatalysts, Chemosphere, 61, 1, 11-18, (2005). 
[43] El-Bahy, Z. M., Ismail, A. A., Mohamed, R. M., Enhancement of titania by doping rare earth for photodegradation of organic dye (Direct Blue), Journal of Hazardous Materials, 166, 1, 138-143, (2009).

[44] Armagan, B., Turan, M. Elik, M. S., Equilibrium studies on the adsorption of reactive azo dyes into zeolite, Desalination, 170, 1, 33-39, (2004).

[45] Ertugay, N., Acar, F. N., Removal of COD and color from Direct Blue 71 azo dye wastewater by Fenton's oxidation: Kinetic study, Arabian Journal of Chemistry, 10, 1, S1158-S1163, (2017).

[46] Hassaan, M. A., El Nemr, A., Madkour, F. F., Testing the advanced oxidation processes on the degradation of Direct Blue 86 dye in wastewater, The Egyptian Journal of Aquatic Research, 43, 1, 11-19, (2017).

[47] Fardood, S. T., Ramazani, A., Moradi, S., Asiabi, P. A., Green synthesis of zinc oxide nanoparticles using arabic gum and photocatalytic degradation of direct blue 129 dye under visible light, Journal of Materials Science: Materials in Electronics, 28, 18, 13596-13601, (2017).

[48] Hefnawy, M. A., Gharieb, M. M., Shaaban, M. T., Soliman, A. M., Optimization of culture condition for enhanced decolorization of Direct Blue dye by Aspergillus flavus and Penicillium canescens, Journal of Applied Pharmaceutical Science, 7, 02, 083-092, (2017).

[49] Esgair, K. K., A study on the removal of Direct Blue 71 dye from textile wastewater produced from state company of cotton industries by electrocoagulation using aluminum electrodes, Journal of Engineering, 2, 23, (2017).

[50] Arica, T. A., Ayas, E., Arica, Y., Magnetic MCM-41 silica particles grafted with poly(glycidylmethacrylate) brush: Modification and application for removal of direct dyes, Microporous and Mesoporous Materials, 243, 164-175, (2017).

[51] Nemr, A.E, Abdelwahab, O., El-Sikaily, A., Khaled, A., Removal of direct blue86 from aqueous solution by new activated carbon developed from orange peel. Journal of Hazardous Materials, 161, 1, 102-110, (2009).

[52] Khani, R., Sobhani, S., Beyki, M. H., Miri, S., Application of magnetic ionomer for development of very fast and highly efficient uptake of triazo dye Direct Blue 71 form different water samples, Ecotoxicology and Environmental Safety, 150, 54-61, (2018).

[53] Fard, R. F., Sar, M. E. K., Fahiminia, M., Mirzaei, N., Yousefi, N., Mansoorian, H. J., Khanjani, N., Rezaei, S., Ghadiri, S. K., Efficiency of multi walled carbon nanotubes for removing Direct Blue 71 from aqueous solutions, Eurasian Journal of Analytical Chemistry, 13, 3, 1-10, (2018).

[54] Hadi, M., Using Thomas model to evaluate dye removal from aqueous solutions in fixed-bed columns of activated carbon, Journal of Water and wastewater, 1, 23-34, (2011).

[55] Bazrafshan, E., Evaluation of color removal of Methylene blue from aqueous solutions using plant stem ash of Persica, Journal of North Khorasan University of Medical Sciences, 4, 4, 523-532, (2011).

[56] Malakootian, M., Fluoride removal using Regenerated Spent Bleaching Earth (RSBE) from groundwater: Case study on Kuhbonan water, Desalination, 277, 1, 244-249, (2011).

[57] Malakootian, M., Evaluating the effectiveness of modified pumice in fluoride removal from water, Asian Journal of Chemistry, 23, 8, 3691, (2011). 
[58] Bulut, Y., Gözübenli, N., Aydın, H., Equilibrium and kinetics studies for adsorption of direct blue 71 from aqueous solution by wheat shells, Journal of Hazardous Materials, 144, 1-2, 300-306, (2007).

[59] Fard, R. F., Kale Sar, M. E., Fahiminia, M., Mirzaei, N., Yousefi, N., Mansoorian, H. J., Khanjani, N., Rezaei, S., Ghadiri, S. K., Efficiency of multi walled carbon nanotubes for removing Direct Blue 71 from aqueous solutions, Eurasian Journal of Analytical Chemistry, 13, 2, 1-10, (2018).

[60] Rehman, R., Mahmud, T., Ejaz, R., Rauf, A., Mitu, L., Sorptive removal of Direct Blue-15 dye from water using Camellia sinensis and Carica papaya leaves, Bulgarian Chemical Communications, 49, 1, 20 - 25, (2017).

[61] Biglari, H., Javan, N., Khosravi, R., Zarei, A., Direct Blue 71 removal from aqueous solutions by adsorption on Pistachio hull waste: Equilibrium, kinetic and thermodynamic studies, Iranian Journal of Health Sciences, 4, 2, 55-70, (2016).

[62] Mirzaei, N., Mahvi, A. H., Hossini, H., Equilibrium and kinetics studies of Direct blue 71 adsorption from aqueous solutions using modified zeolite, Adsorption Science \& Technology, 36, 1-2, 80-94 (2018).

[63] Prola, L. D. T., Machado, F. M., Bergmann, C. P., de Souza, F. E., Gally, C. R., Lima, C., Adebayo, M. A., Dias, S. L. P., Calvete, T., Adsorption of Direct Blue 53 dye from aqueous solutions by multi-walled carbon nanotubes and activated carbon, Journal of Environmental Management, 130, 166-175, (2013).

[64] Nguyen, V. H., Haldorai, Y. ve Shim, J. J., Supercritical fluid mediated synthesis of poly(2-hydroxyethyl methacrylate)/ $/ \mathrm{Fe}_{3} \mathrm{O}_{4}$ hybrid nanocomposite, Materials Science and Engineering: B, 176, 773-778, (2011).

[65] Nguyen, V. H. ve Shim, J. J., Supercritical fluid-assisted synthesis of a carbon nanotubes-grafted biocompatible polymer composite, Composite Interfaces, 20, 155-162, (2013).

[66] Kharismadewi, D., Haldorai, Y., Nguyen, V. H., Tuma, D. ve Shim, J. J., Synthesis of graphene oxide-poly(2-hydroxyethyl methacrylate) composite by dispersion polymerization in supercritical $\mathrm{CO}_{2}$ : adsorption behavior for the removal of organic dye, Composite Interfaces, 23, 7, 719-739, (2016).

[67] Özer, E. T., Göçenoğlu Sarıkaya, A. ve Osman, B., Adsorption and removal of diethyl phthalate from aqueous media with poly(hydroxyethyl methacrylate) nanobeads, Desalination and Water Treatmant, 57, 59, 28864-28874, (2016).

[68] Langmuir, I., The adsorption of gases on plane surfaces of glass, mica and platinum, Journal of American Chemical Society, 40, 1361-1403, (1918).

[69] El-Halwany, M. M., Study of adsorption isotherms and kinetic models for Methylene Blue adsorption on activated carbon developed from Egyptian rice hull (Part II), Desalination, 250, 208-213, (2010).

[70] Freundlich H., Over the adsorption in solution, The Journal of Physical Chemistry, 57, 385, (1906).

[71] Özüdoğru, Y. ve Merdivan, M., Metilen mavisinin modifiye edilmiş Cystoseira barbata (stackhouse) c. agardh kullanılarak biyosorpsiyonu, Trakya University Journal of Natural Sciences, 18, 2, 81-87, (2017).

[72] Foo, K. Y. ve Hameed, B. H., Insights into the modeling of adsorption isotherm systems, Chemical Engineering Journal, 156, 1, 2-10, (2010).

[73] Wang, S., Boyjoo, Y. ve Choueib, A. A., Comparative study of dye removal using fly ash treated by different methods, Chemosphere, 60, 10, 1401-1407, (2005) 
[74] Biglari, H., Javan, N., Khosravi, R. ve Zarei, A., Direct blue 71 removal from aqueous solutions by adsorption on pistachio hull waste: equilibrium, kinetic and thermodynamic studies, Iranian Journal of Health Sciences, 4, 2, 55-70, (2016).

[75] Adak, A., Bandyopadhyay, M., Pal, A, Removal of crystal violet dye from wastewater by surfactant-modified alümina, Separation and Purification Technology, 44, 2, 139-144, (2005).

[76] Weng, C. H. ve Pan, Y. F, Adsorption of a cationic dye (methylene blue) onto spent activated clay, The Journal of Hazardous Materials, 144, 1, 355-362, (2007).

[77] Nandi, B. K., Goswami, A., Das, A. K., Mondal, B., Purkait, M. K., Kinetic and equilibrium studies on the adsorption of crystal violet dye using kaolin as an adsorbent, Separation Science and Technology, 43, 6, 1382-1403, (2008).

[78] Kumar, K.V., Ramamurthi, V., Sivanesan, S., Modeling the mechanism involved during the sorption of methylene blue onto fly ash, Journal of Colloid and Interface Science, 284, 1, 14-21, (2005).

[79] Yagub, M.T., Sen, T.K., Ang, H., Equilibrium, kinetics, and thermodynamics of methylene blue adsorption by pine tree leaves, Water Air Soil Pollution, 223, 8, 5267-5282, (2012).

[80] Nassar, N. N., Kinetics, mechanistic, equilibrium, and thermodynamic studies on the adsorption of acid red dye from wastewater by $\gamma-\mathrm{Fe}_{2} \mathrm{O}_{3}$ nanoadsorbents, Separation Science and Technology, 45, 8, 1092-1103, (2010).

[81] Khan, T. ve Chaudhuri, M., Adsorptive removal of Direct Blue 86 by coconut coir activated carbon, Environmental Science and Technology Conference (ESTEC2009), Kuala Terengganu, Malaysia, (2009).

[82] Kumar A., Saakshy, Vyas R. K., Adsorption of direct blue 5 dye by activated carbon as adsorbent- modeling and kinetics, International Journal of Engineering Research\&Technology (IJERT), 2, 12, 2246-2255, (2013).

[83] Kanchi, S., Bisetty, K., Kumar, G., Robust adsorption of Direct Navy Blue-106 from textile industrial effluents by bio-hydrogen fermented waste derived activated carbon: Equilibrium and kinetic studies, Arabian Journal of Chemistry, 10, 2) S3084-S3096, (2017).

[84] Pathania, D., Sharma, S., Singh, P., Removal of methylene blue by adsorption onto activated carbon developed from Ficus carica bast, Arabian Journal of Chemistry, 10, 1, S1445-S1451, (2017).

[85] Abbasi, M., Synthesis and characterization of magnetic nanocomposite of chitosan/SiO2/carbon nanotubes and its application for dyes removal, Journal of Cleaner Production, 145, 105-113, (2017).

[86] Zarei, M., Djafarzadeh, N., Khadir, L., Removal of direct blue 129 from aqueous medium using surfactant-modified zeolite: a neural network modeling, Environmental Health Engineering and Management Journal, 5, 2, 101113, (2018).

[87] Miranda-Mandujano, E., Moeller-Chávez, G., Villegas-Rosas, O., Buitrón, G., Garzón-Zúñiga, M. A., Decolourization of Direct Blue 2 by peroxidases obtained from an industrial soybean waste, Water SA, 44, 2, 204-210, (2018).

[88] Mouni, L., Belkhiri, L., Bollinger, J. C., Bouzaza, A., Assadi, A., Tirri, A. Dahmoune, F., Madani, K., Remini, H., Removal of Methylene Blue from aqueous solutions by adsorption on kaolin: Kinetic and equilibrium studies, Applied Clay Science, 153, 38-45, (2018). 
[89] Aksu, Z. ve Çağatay, Ş. Ş., Investigation of biosorption of Gemazol Turquise Blue-G reactive dye by dried Rhizopus arrhizus in batch and continuous systems, Separation and Purification Technology, 48, 1, 24-35, (2006).

[90] Mane, V. S., Mall, I. D. ve Srivastava, V. C., Kinetic and equilibrium isotherm studies for the adsorptive removal of Brilliant Green dye from aqueous solution by rice husk ash, Journal of Environmental Management, 84, 390-400, (2007). 